\title{
Endocytosis of Multiwalled Carbon Nanotubes in Bronchial Epithelial and Mesothelial Cells
}

\author{
Kayo Maruyama, ${ }^{1}$ Hisao Haniu, ${ }^{1,2}$ Naoto Saito, ${ }^{1}$ Yoshikazu Matsuda, ${ }^{3}$ \\ Tamotsu Tsukahara, ${ }^{4}$ Shinsuke Kobayashi, ${ }^{2}$ Manabu Tanaka, ${ }^{2}$ Kaoru Aoki, ${ }^{2}$ \\ Seiji Takanashi, ${ }^{2}$ Masanori Okamoto, ${ }^{2}$ and Hiroyuki Kato ${ }^{2}$ \\ ${ }^{1}$ Institute for Biomedical Sciences, Interdisciplinary Cluster for Cutting Edge Research, Shinshu University, 3-1-1 Asahi, \\ Matsumoto, Nagano 390-8621, Japan \\ ${ }^{2}$ Department of Orthopaedic Surgery, Shinshu University School of Medicine, 3-1-1 Asahi, Matsumoto, Nagano 390-8621, Japan \\ ${ }^{3}$ Clinical Pharmacology Educational Center, Nihon Pharmaceutical University, 10281 Komuro, Ina-machi, Saitama 362-0806, Japan \\ ${ }^{4}$ Department of Molecular Pharmacology and Neuroscience, Nagasaki University Graduate School of Biomedical Sciences, \\ 1-14 Bunkyo-machi, Nagasaki 852-8521, Japan
}

Correspondence should be addressed to Hisao Haniu; hhaniu@shinshu-u.ac.jp

Received 27 June 2014; Accepted 26 December 2014

Academic Editor: Haseeb A. Khan

Copyright ( 2015 Kayo Maruyama et al. This is an open access article distributed under the Creative Commons Attribution License, which permits unrestricted use, distribution, and reproduction in any medium, provided the original work is properly cited.

\begin{abstract}
Bronchial epithelial cells and mesothelial cells are crucial targets for the safety assessment of inhalation of carbon nanotubes (CNTs), which resemble asbestos particles in shape. Intrinsic properties of multiwalled CNTs (MWCNTs) are known to cause potentially hazardous effects on intracellular and extracellular pathways. These interactions alter cellular signaling and affect major cell functions, resulting in cell death, lysosome injury, reactive oxygen species production, apoptosis, and cytokine release. Furthermore, CNTs are emerging as a novel class of autophagy inducers. Thus, in this study, we focused on the mechanisms of MWCNT uptake into the human bronchial epithelial cells (HBECs) and human mesothelial cells (HMCs). We verified that MWCNTs are actively internalized into HBECs and HMCs and were accumulated in the lysosomes of the cells after 24-hour treatment. Next, we determined which endocytosis pathways (clathrin-mediated, caveolae-mediated, and macropinocytosis) were associated with MWCNT internalization by using corresponding endocytosis inhibitors, in two nonphagocytic cell lines derived from bronchial epithelial cells and mesothelioma cells. Clathrin-mediated endocytosis inhibitors significantly suppressed MWCNT uptake, whereas caveolae-mediated endocytosis and macropinocytosis were also found to be involved in MWCNT uptake. Thus, MWCNTs were positively taken up by nonphagocytic cells, and their cytotoxicity was closely related to these three endocytosis pathways.
\end{abstract}

\section{Introduction}

Carbon nanotubes (CNTs) were first discovered by Oberlin et al. [1], and they have attracted increasing attention since the end of 20th century. Owing to their unique physical, mechanical, and electronic properties, CNTs serve as valuable reinforcements or enhance the properties and introduce novel functionalities of various materials in a number of fields, including chemistry, electronics, energy, and materials science $[2,3]$. The unique properties of CNTs have also garnered considerable attention from the fields of medicine and biology, and they have potential applications as biomaterials for biosensors, drug and vaccine delivery vehicles, and scaffold materials [4-6].

However, the potential adverse effects of CNTs on human health are of great concern, considering their increasing use in composite biomaterials and exploration as innovative solutions for biomedical applications or in nanomedicine as well as the potential workplace exposure [7-9]. CNTs possess asbestos-like morphological characteristics (i.e., a nanoscale size and a high aspect ratio) and persist in the human body for a long time [10-12]. In 2008, Takagi et al. reported that 
transgenic mice intraperitoneally injected with MWCNTs exhibited mesothelioma similar to that in mice exposed to asbestos [13]. Subsequently, induction of mesothelioma was also reported after intraperitoneal or intrascrotal injection of CNTs in rodents [14-16]. Moreover, some evidence suggests that CNT causes cancer upon inhalation or intratracheal administration [17-20], although there is no direct evidence that CNTs induce pleural mesothelioma and lung cancer [17, 21-24].

Previous studies have clarified the carcinogenic mechanisms of CNTs in vitro. The number of micronuclei in lung epithelial cells increases upon exposure to MWCNTs, which is indicative of genotoxicity such as chromosomal damage or mitotic spindle disruption [20]. Sargent et al. showed that CNTs induce mitotic spindle disruption that results in errors in chromosome number $[8,25-27]$. CNTs must be internalized by cells for such phenomena to occur. We have previously reported that it is important for multiwalled CNTs (MWCNTs) to be internalized for cytotoxic effects to be observed in a human mesothelioma cell line (MESO-1) and a human bronchial epithelial cell line (BEAS-2B) [28-30]. However, the internalization mechanism of CNTs is not well known.

In this study, we demonstrated the mechanism underlying CNT internalization in human primary bronchial epithelial cells and mesothelium cells. Moreover, we also demonstrated the internalization mechanism of CNTs in nonphagocytic cells by using various endocytosis inhibitors.

\section{Materials and Methods}

2.1. Carbon Nanotubes. MWCNTs manufactured by a chemical vapor deposition method [31] were provided by Hodogaya Chemical (MWNT-7; Tokyo, Japan); their properties have been reported previously [32]. The sterilization conditions were autoclaving at $121^{\circ} \mathrm{C}$ for $15 \mathrm{~min}$. MWCNTs were vortexed for $1 \mathrm{~min}$ in $0.1 \%$ gelatin (MediGelatin; Nippi, Tokyo, Japan) or $2 \%$ fetal bovine serum (FBS; Life Technologies, Grand Island, NY, USA) in phosphate-buffered saline (PBS) and sonicated for $30 \mathrm{~min}$. MWNT-7 was diluted if required, and a volume of 1/100 was added to the cell culture fluid in the following exposure experiments.

2.2. Endocytosis Inhibitors. The endocytosis inhibitors used were previously described by Yumoto et al. [33]. Phenylarsine oxide, indomethacin, nystatin, and 5-(N-ethyl-N-isopropyl)amiloride were purchased from Sigma-Aldrich (St. Louis, MO, USA). Chlorpromazine was purchased from Nacalai Tesque (Kyoto, Japan). Phenylarsine oxide was dissolved in dimethyl sulfoxide (DMSO) and diluted to $0.2-5 \mathrm{mM}$. Indomethacin was dissolved in ethanol at $50^{\circ} \mathrm{C}$ and diluted to 5$100 \mathrm{mM}$. Nystatin was dissolved in DMSO and diluted to 120 mM. 5-(N-Ethyl-N-isopropyl)amiloride was dissolved in DMSO and diluted to 5-80 mM. Chlorpromazine was dissolved in PBS and diluted to $2-50 \mathrm{mM}$.

2.3. Cell Culture. Normal human bronchial epithelial cells (HBECs) were purchased from Lonza (Walkersville, MD,
USA). Normal human mesothelial cells (HMCs) were purchased from Zen-Bio, Inc. (Research Triangle Park, NC, USA). The BEAS-2B human bronchial epithelial cell line was purchased from American Type Culture Collection (Manassas, VA, USA). The ACC-MESO-1 human malignant pleural mesothelioma cell line [34] was purchased from RIKEN (Ibaraki, Japan). HBECs were cultured in bronchial/tracheal epithelial cell serum-free growth medium kit with $0.1 \mu \mathrm{g} / \mathrm{mL}$ retinoic acid (Cell Application, San Diego, CA, USA) and passaged every $4 \mathrm{~d}$, with the medium exchanged every alternate day. HMCs were cultured in mesothelial cell growth medium (Zen-Bio, Inc.) and passaged twice a week. Both types of normal cell were used within 5 passages. BEAS-2B cells were cultured in Ham's nutrient mixture F-12 (Nacalai Tesque) with $10 \%$ FBS and passaged twice a week. MESO-1 cells were cultured in RPMI 1640 (Nacalai Tesque) with 10\% FBS and passaged twice a week. For each experiment, the cells were seeded at a density of $2 \times 10^{5}$ cells $/ \mathrm{cm}^{2}$ and allowed to adhere for $24 \mathrm{~h}$.

2.4. Cell Viability. The cell viability assay was performed as described previously [35]. We performed an Alamar Blue assay (alamarBlue cell viability reagent; Invitrogen, Carlsbad, CA) according to the manufacturer's instructions. Cells were plated in 96-well plates and incubated for $24 \mathrm{~h}$ at $37^{\circ} \mathrm{C}$ in the culture medium containing MWCNTs in a dispersant or in a control medium containing only dispersant without MWCNTs. Viable cells metabolized the dye, resulting in increased fluorescence detected by excitation/emission at 530/590 nm using a fluorescence multiplate reader (PowerScan 4; DS Pharma Biomedical, Osaka, Japan). Cell viability was calculated as follows: percent cytotoxicity $=100 \times$ experimental value/control value. The test media were assayed six times for each treatment condition.

2.5. Imaging of MWNT-7 Uptake by Fluorescence Microscopy. Cells were cultured on ibiTreat $\mu$-Slide (ibidi GmbH, Martinsried, Germany) for snapshot imaging and ibiTreat $\mu$-dish for time-lapse imaging for $24 \mathrm{~h}$ in a $5 \% \mathrm{CO}_{2}$ incubator. The cells were prestained with bisbenzimide H33342 fluorochrome trihydrochloride (H33342, $1 \mu \mathrm{g} / \mathrm{mL}$; Nacalai) and CytoPainter Lysosomal Staining Kit (Abcam, Tokyo, Japan) for $2 \mathrm{~h}$. Then, the cells were washed once and exposed to MWNT$7(10 \mu \mathrm{g} / \mathrm{mL})$. MWNT-7 uptake was snapshot-imaged at 2, 6, and $24 \mathrm{~h}$, and time-lapse imaging was performed at $10 \mathrm{~min}$ intervals for $24 \mathrm{~h}$ by using differential interference contrast (DIC) and fluorescence imaging by fluorescence microscopy with cell culture equipment (AxioObserver Z1, Zeiss, Jena, Germany) using a 40x objective.

2.6. Assessment of MWNT-7 Uptake by Flow Cytometry. Cells were cultured on a 12 -well plate for $24 \mathrm{~h}$ in a $5 \% \mathrm{CO}_{2}$ incubator. Endocytosis inhibitors were pretreated $15 \mathrm{~min}$ before CNT exposure. Then, the cells were exposed to MWNT-7 $(10 \mu \mathrm{g} / \mathrm{mL})$ and incubated for $2 \mathrm{~h}$. The evaluation of cellular uptake for MWNT-7 followed the method reported that we reported previously [28]. In brief, the cells treated with or without MWNT-7 were washed twice and trypsinized. 


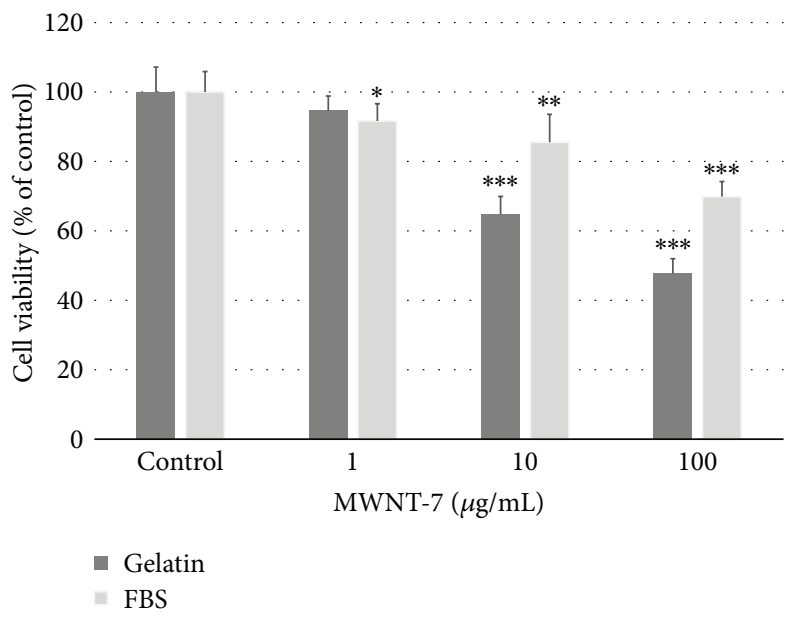

(a)

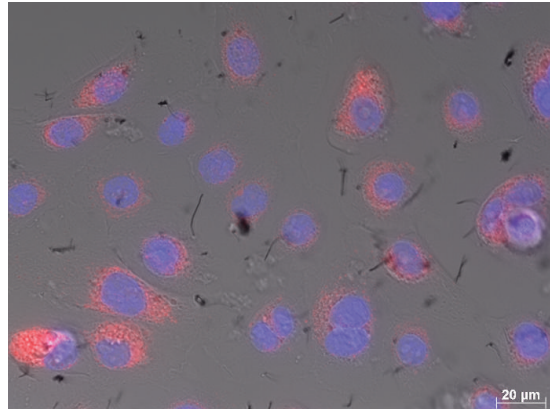

(b)

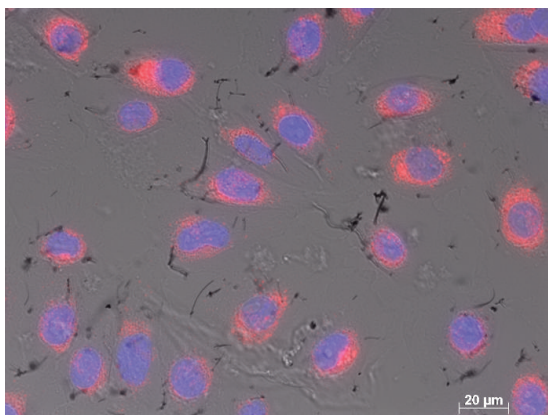

(e)

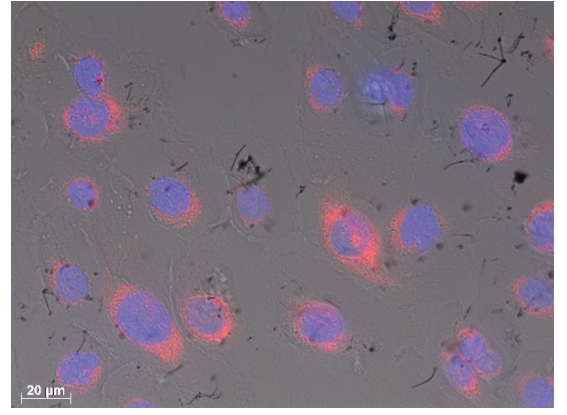

(c)

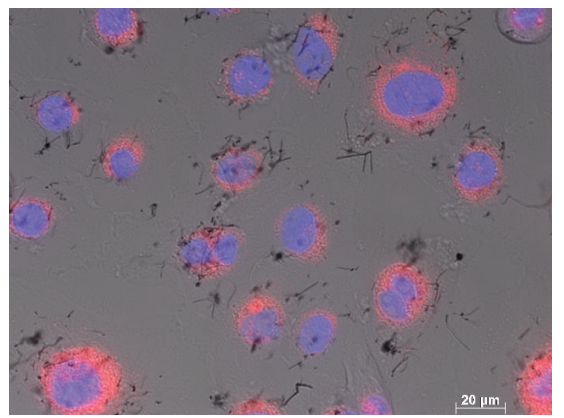

(f)

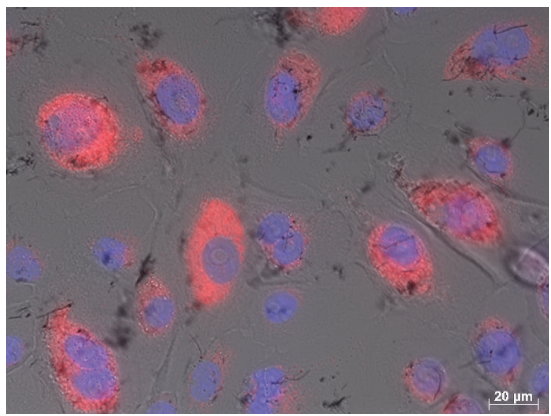

(d)

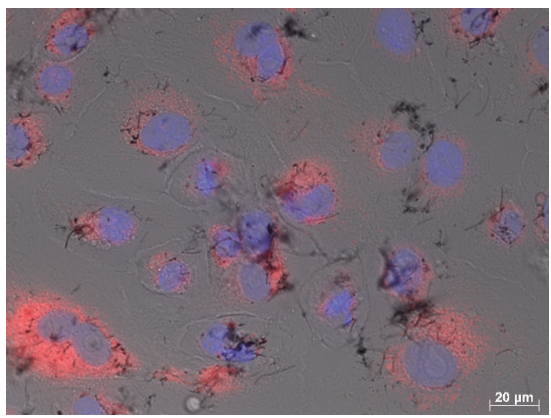

(g)

Figure 1: HBECs were exposed to MWNT-7. (a) Viability of HBECs exposed to various concentrations of MWNT-7 in $0.1 \%$ gelatin or $2 \%$ FBS for $24 \mathrm{~h}$. HMCs were compared with HBECs exposed to MWNT-7 in each type of dispersant and to the control. Mean \pm SD. $n=6$, ${ }^{*} P<0.05,{ }^{* *} P<0.01$, and ${ }^{* * *} P<0.001$. Image of HBECs exposed to $10 \mu \mathrm{g} / \mathrm{mL}$ MWNT-7 in $0.1 \%$ gelatin at $2 \mathrm{~h}(\mathrm{~b}), 6 \mathrm{~h}(\mathrm{c})$, and $24 \mathrm{~h}(\mathrm{~d})$ and in $2 \%$ FBS at $2 \mathrm{~h}(\mathrm{e}), 6 \mathrm{~h}(\mathrm{f})$, and $24 \mathrm{~h}(\mathrm{~g})$. DIC and fluorescence images were merged. Nuclei were stained blue with $\mathrm{H} 33342$ and lysosomes were stained red with CytoPainter.

The cells suspended in PBS containing 10\% FBS were filtered through a nylon mesh. Then, the cells were assayed for side scatter (SSC) by light scattering analysis using a flow cytometer (FCM; FACSCalibur, Becton Dickinson, San Jose, CA, USA). The SSC ratio was calculated by dividing the MWNT-7 value with the control value.

2.7. Statistical Analysis. Data are presented as the mean \pm standard deviation (SD). Statistical significance was determined by analysis of variance (ANOVA) followed by Student's $t$-test, and $P<0.05$ was considered to be significant.

\section{Results and Discussion}

3.1. Cellular Uptake by HBECs and HMCs. First, we determined whether CNTs could be internalized in normal human bronchial epithelial and mesothelial cells, for which potential carcinogenicity of CNTs is of concern. Although we had already shown that human mesothelioma cells and commercialized normal HBECs from Cell Application internalized CNTs $[28,32,36]$, Nagai et al. reported that normal human primary cultured mesothelium cells did not internalize CNTs [16]. We used HBECs purchased from a different company 


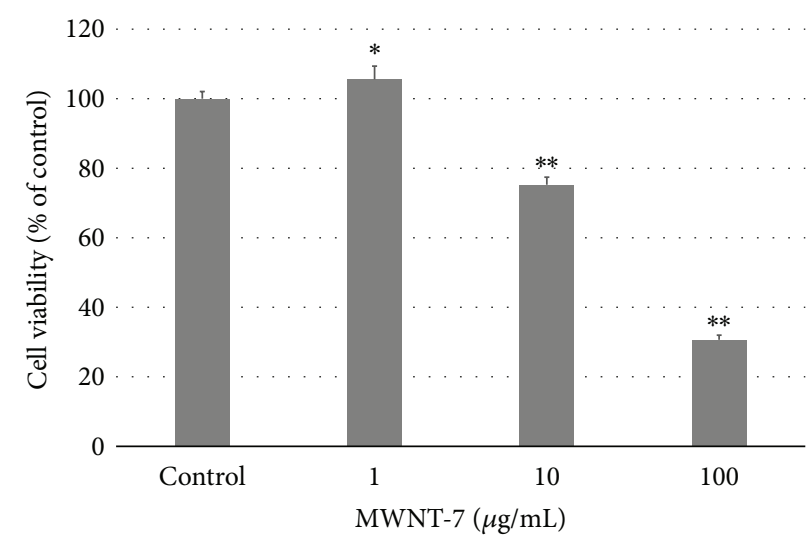

(a)

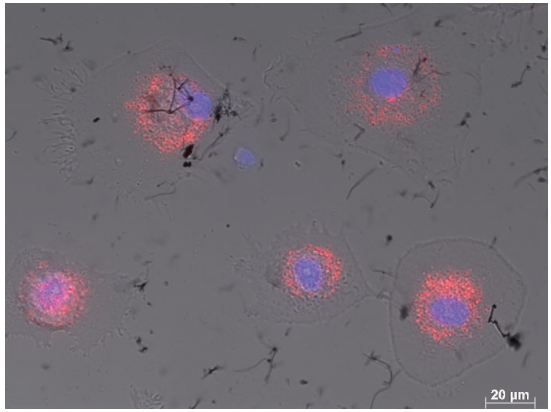

(b)

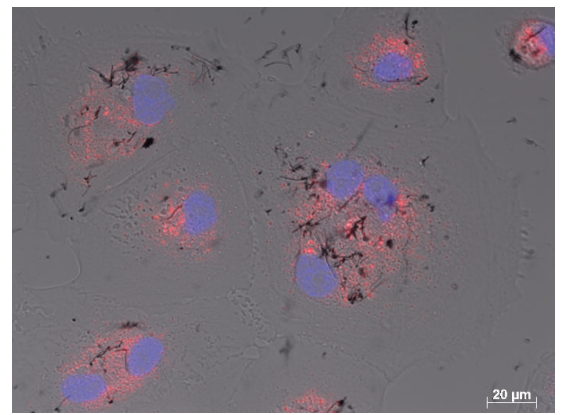

(c)

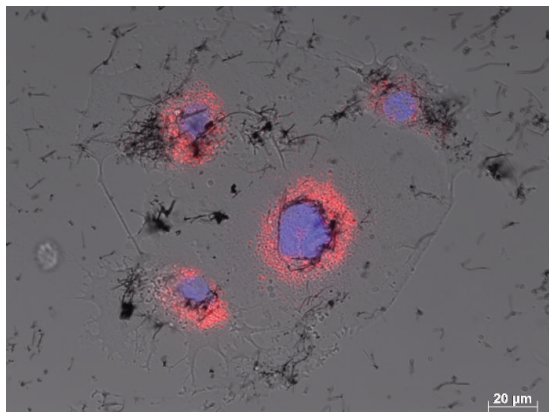

(d)

FIGURE 2: HMCs were exposed to MWNT-7. (a) Viability of HMCs exposed to variety concentration of MWNT-7 in $0.1 \%$ gelatin for 24 h. $P$ values were compared to HMCs exposed to MWNT-7 in dispersant control. Mean \pm S.D. $n=6,{ }^{*} P<0.05$, ${ }^{* *} P<0.001$. Image of HMCs exposed to $10 \mu \mathrm{g} / \mathrm{mL}$ MWNT-7 in $0.1 \%$ gelatin at $2 \mathrm{~h}(\mathrm{~b}), 6 \mathrm{~h}(\mathrm{c})$, and $24 \mathrm{~h}(\mathrm{~d})$. DIC and fluorescence images were merged. Nuclei were stained blue with $\mathrm{H} 33342$ and lysosome were stained red with CytoPainter.

from that used in the previous paper to evaluate the influence of supplier on cellular uptake of CNTs. Moreover, we compared FBS as a dispersant for CNTs with gelatin in HBECs because the dispersion of CNTs by $2 \%$ FBS was recommended by the ENPRA [37]. The viability of HBECs from Lonza at $10 \mu \mathrm{g} / \mathrm{mL}$ MWNT-7 for $24 \mathrm{~h}$ was approximately $85.5 \%$ in FBS and $64.7 \%$ in gelatin, and the cell viability decreased at higher concentrations $(100 \mu \mathrm{g} / \mathrm{mL})$ in both dispersants in a concentration-dependent manner (69.9\% versus $47.7 \%$; Figure 1(a)). We observed cells dyed with fluorescence to determine whether CNTs were internalized in the cells. The visualized cells began to internalize MWNT-7 dispersed in not only gelatin but also FBS within $2 \mathrm{~h}$ in some cells, and uptake of MWNT-7 was observed in most cells within $6 \mathrm{~h}$ (Figures 1(b), 1(c), 1(e), and 1(f)). At $24 \mathrm{~h}$, MWNT-7 appeared to accumulate in lysosomes (Figures $1(\mathrm{~d})$ and $1(\mathrm{~g})$ ). Because the purpose of this paper was to elucidate the mechanisms underlying the endocytosis of CNTs, the CNTs used in subsequent experiments were dispersed with gelatin to prevent the influence of unknown factors.

Although the viability of HMCs exposed to MWNT-7 dispersed in gelatin decreased in a concentration-dependent manner (Figure 2(a)) the cell viability was still higher than that of HBECs. HMCs also began to internalize MWNT7 within $2 \mathrm{~h}$, and the internalization of MWNT-7 increased over time (Figures 2(b)-2(d)). We previously reported that BEAS-2B cells derived from human bronchial epithelium and MESO-1 cells derived from human malignant mesothelioma showed cytotoxicity arising from lysosomal injury [35]. Human normal bronchial epithelial and mesothelial cells also showed MWNT-7 internalization and cytotoxicity dependent on MWNT-7, which accumulated in the lysosome in excessive concentrations. Although Nagai et al. found that human primary mesothelium cells exposed to MWCNTs did not internalize the MWCNTs based on the SSC ratio, transmission electron microscopy, confocal microscopy, and time-lapse microscopy, they evaluated the results at $3 \mathrm{~h}$ after exposure to the materials [16]. We speculate that these results were obtained because it is difficult for CNTs to sink in the solution owing to their very light weight. In fact, our results showed that uptake of MWNT-7 observed by DIC increased over time, and only a few cells internalized MWNT-7 in $2 \mathrm{~h}$. It has also been reported that the quantity of CNTs that undergo cellular uptake increases until approximately $12 \mathrm{~h}$ [38]. Moreover, although previous studies have evaluated the uptake of CNTs in comparison with asbestos, such a comparison under the same conditions is not effective because cellular uptake of different materials depends on their physicochemical properties. Another study showed that MWCNT exerted adverse effects without CNT uptake in a human mesothelial cell line 


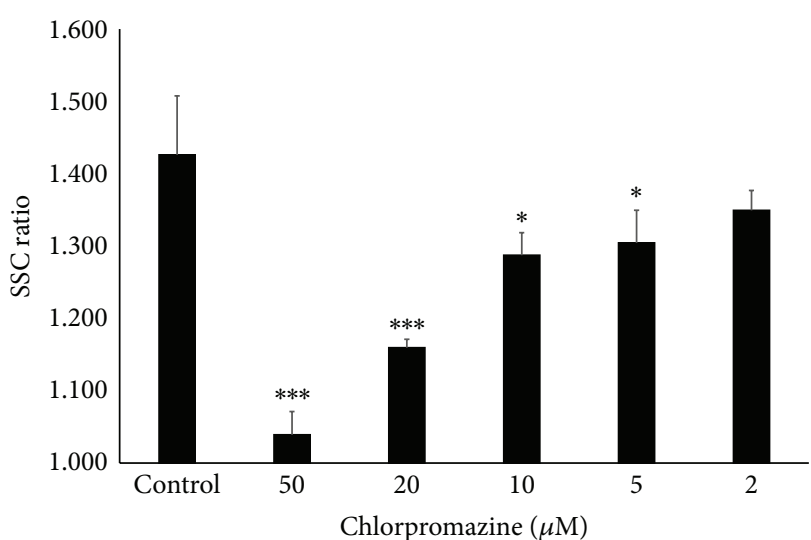

(a)

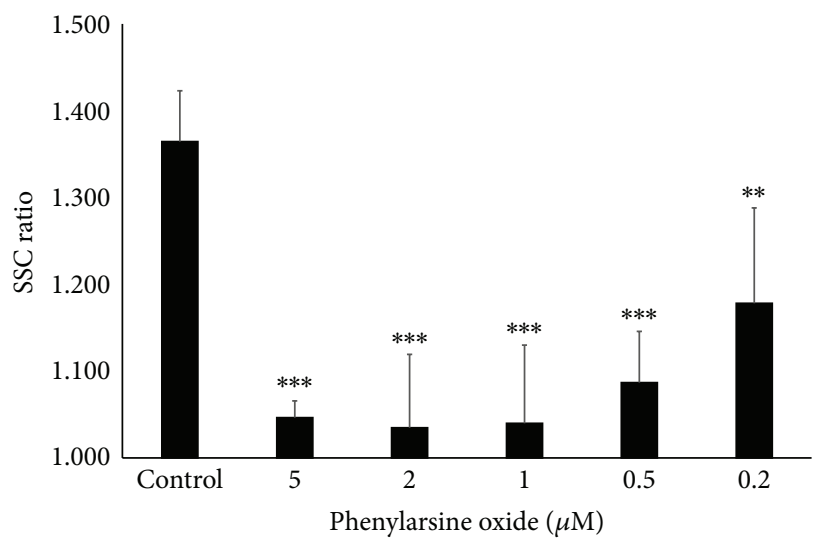

(c)

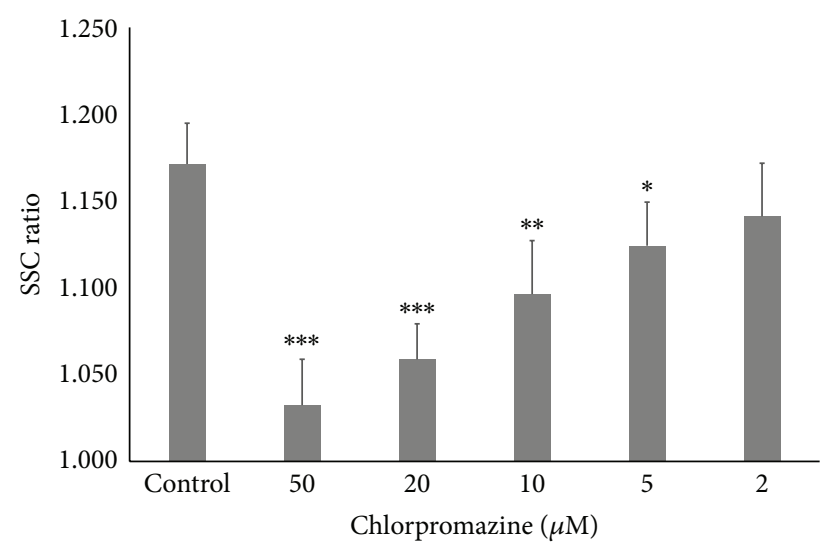

(b)

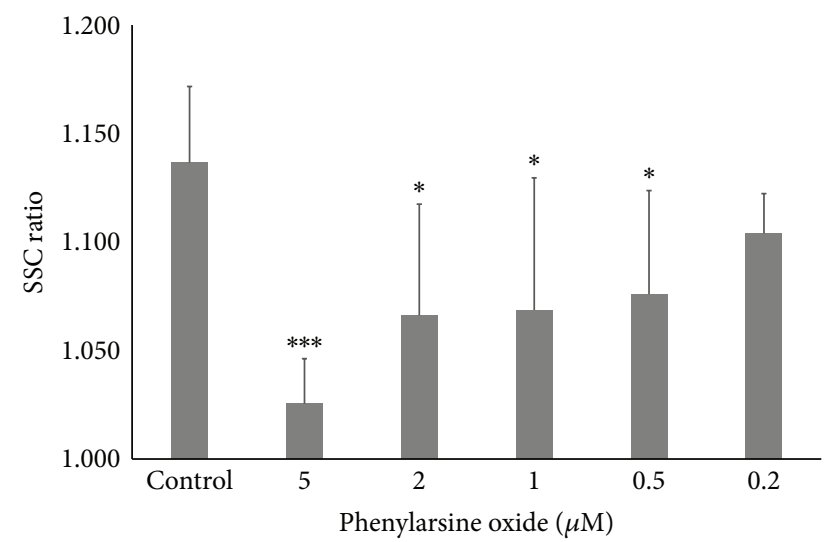

(d)

FIGURE 3: Effect of clathrin-mediated endocytosis inhibitors on cellular uptake of MWNT-7. The SSC ratios of (a) BEAS-2B cells and (b) MESO-1 cells pretreated with various concentrations of chlorpromazine are shown. The SSC ratios of (c) BEAS-2B cells and (d) MESO-1 cells pretreated with various concentrations of phenylarsine oxide are shown. The cells were compared with control cells pretreated with inhibitor solvent. Mean \pm SD. $n=4$ or $6,{ }^{*} P<0.05,{ }^{* *} P<0.01$, and ${ }^{* * *} P<0.001$.

(Met-5A) [39]. However, although they showed transmission electron microscopy (TEM) images of A549 alveolar epithelial cell lines to conclude that the cells do not internalize CNTs, no TEM images for Met-5A were presented, and optical microscope images of low magnification $(\times 20)$ were only shown. Lindberg et al. reported genotoxicity of MWCNTs based on TEM images showing that the Met-5A cells internalize CNTs [40]. Moreover, our time-lapse data clearly and directly indicate that HBECs and HMCs endocytose MWCNTs actively (Movies S1 and S2) (see Supplementary Material available online at http://dx.doi.org/10.1155/2015/793186). We also have already reported that the BEAS-2B cell line derived from human bronchial cells and MESO-1 cells derived from human mesothelioma cells internalized some MWCNTs [28, 36]. Therefore, we used inhibitors of endocytosis, to clarify the internalization mechanism of CNT further using BEAS$2 \mathrm{~B}$ cells and MESO- 1 cells rather than HBECs and HMCs, respectively.

We investigated the mechanism of CNT uptake using inhibitors for three endocytosis pathways (clathrin-mediated, caveolae-mediated, and macropinocytosis), with the SSC ratio as an index. We have already shown that SSC ratio increases concentration dependently over time in cells that only internalized CNTs [28]. The SSC ratios of the control, which was not pretreated by inhibitors in BEAS- $2 \mathrm{~B}$ and MESO- 1 cells, were $1.355-1.426$ and $1.137-1.258$ in $2 \mathrm{~h}$, respectively. It was observed that the SD of the SSC ratios tended to increase with cell passage number, likely because we analyzed under sixteen passages for both cell lines. Therefore, few statistically significant differences were noted when we assayed the SSC ratios of nystatin as a caveolae-mediated endocytosis inhibitor and 5-(N-ethyl-N-isopropyl)amiloride as a macropinocytosis inhibitor.

Two clathrin-mediated endocytosis inhibitors suppressed the ratio in a concentration-dependent manner in both cell lines (Figures 3(a)-3(d)). In BEAS-2B cells, the maximum concentration of chlorpromazine $(50 \mu \mathrm{M})$ decreased the SSC ratio to 1.039 , whereas the SSC ratio with $2 \mu \mathrm{M}$ phenylarsine oxide was 1.040. In MESO-1 cells, the lowest SSC ratios were 1.032 and 1.025 with treatment with $50 \mu \mathrm{M}$ chlorpromazine and $5 \mu \mathrm{M}$ phenylarsine oxide, respectively. Because the baseline SSC ratio for which the cells were not exposed to CNTs was 1.000 , clathrin-mediated endocytosis seems to be the main mechanism for cellular uptake. 


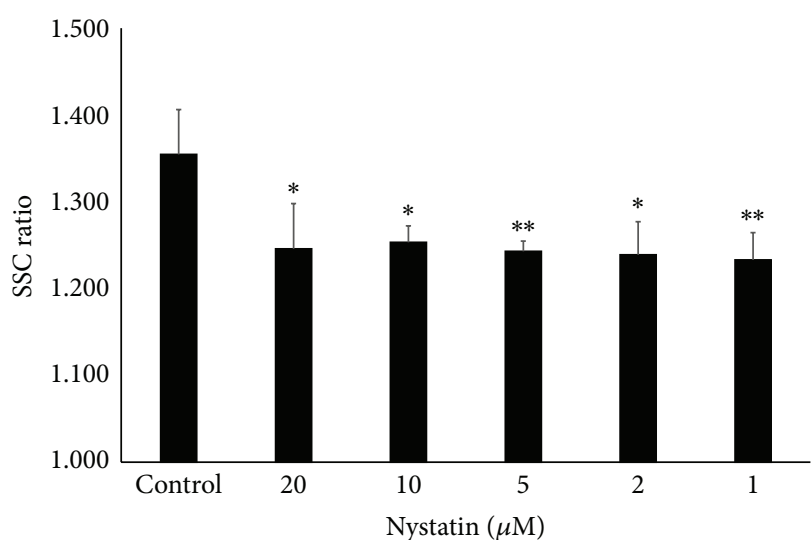

(a)

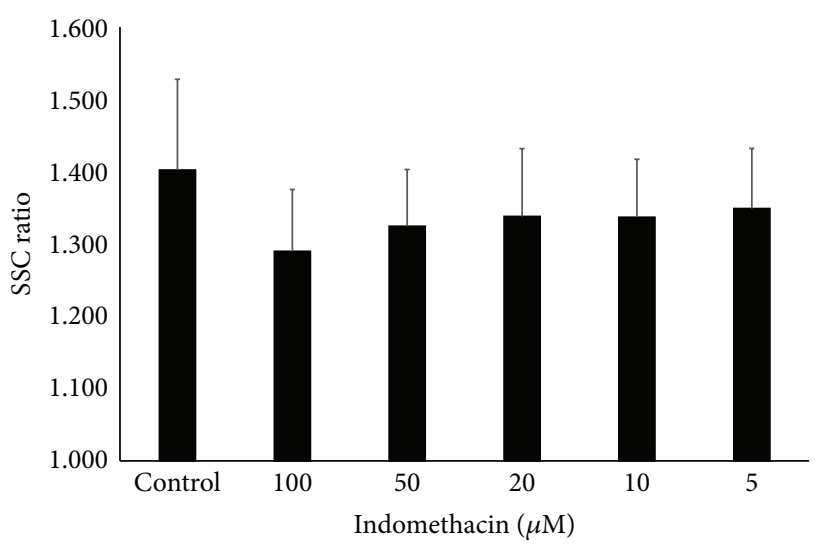

(c)

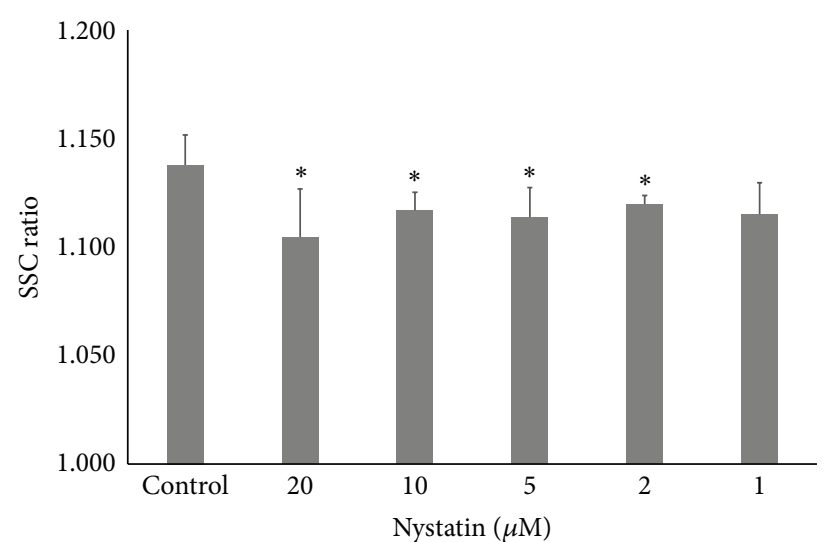

(b)

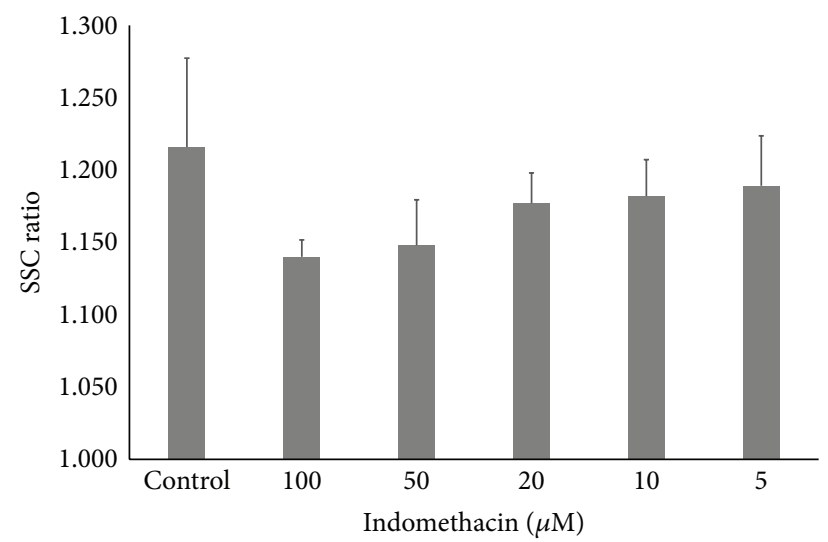

(d)

FIGURE 4: Effect of caveolae-mediated endocytosis inhibitors on cellular uptake of MWNT-7. The SSC ratios of (a) BEAS-2B cells and (b) MESO-1 cells pretreated with various concentrations of nystatin are shown. The SSC ratios of (c) BEAS-2B cells and (d) MESO-1 cells pretreated with the various concentrations of indomethacin are shown. The cells were compared with control cells pretreated with inhibitor solvent. Mean \pm S.D. $n=4,{ }^{*} P<0.05,{ }^{* *} P<0.01$.

The results for caveolae-mediated endocytosis inhibitors were complicated. Nystatin decreased the SSC ratio in both cells significantly except at $1 \mu \mathrm{M}$ in MESO-1 cells (Figures 4(a) and 4(b)). In detail, MESO-1 cells displayed a tendency for concentration dependency, whereas the inhibition did not depend on the dose in BEAS-2B cells. In contrast, although indomethacin tended to show concentration-dependent inhibition in both cell lines, there was no statistically significant difference (Figures 4(c) and 4(d)). The difference in the results may be caused by the inhibition mechanism. Nystatin disrupts caveolar function and binds to sterol in the plasma membrane [41-43]; indomethacin blocks the internalization of caveolae and the return of plasmalemmal vesicles [44, 45]. However, we considered that caveolae-mediated endocytosis pathway may partially contribute to the internalization of CNTs for the following reasons: (1) the inhibition rate of nystatin, which was not concentration-dependent, was $30.7 \%$ and was the same as the inhibition rate $(27.9 \%)$ with the highest concentration of indomethacin $(100 \mu \mathrm{M})$ in BEAS2B cells. (2) In MESO-1 cells, both inhibitors showed a tendency for concentration-dependence, and the inhibition rate provided by indomethacin, which inhibits the essential parts of the endocytosis pathway, was higher than that by nystatin $(35.2 \%$ and $23.9 \%$, resp.). The inhibition of statin binding to the sterol may have been responsible for difference among cell types.

5-(N-Ethyl-N-isopropyl)amiloride, which inhibits the macropinocytosis pathway, seems to suppress CNT uptake in a concentration-dependent manner, although the difference did not reach significance except at $80 \mu \mathrm{M}$ in MESO-1 cells (Figures 5(a) and 5(b)). The inhibition rates of BEAS-2B cells and MESO- 1 cells were comparable at $42.7 \%$ and $56.6 \%$ at the highest concentration $(80 \mu \mathrm{M})$. The role of macropinocytosis in CNT uptake has not been extensively studied. Hirano et al. demonstrated that macrophage receptor with collagenous structure- (MARCO-) transfected CHO-K1 cells takes up MWCNTs via membrane ruffling in a process similar to macropinocytosis [46]. They also reported that MARCO was absorbed in MWCNTs to which macrophages were exposed [47]. However, it was not clear whether macropinocytosis for CNTs occurs in nonphagocytic cells. Our results indicate that macropinocytosis plays an important role in CNTs uptake. 


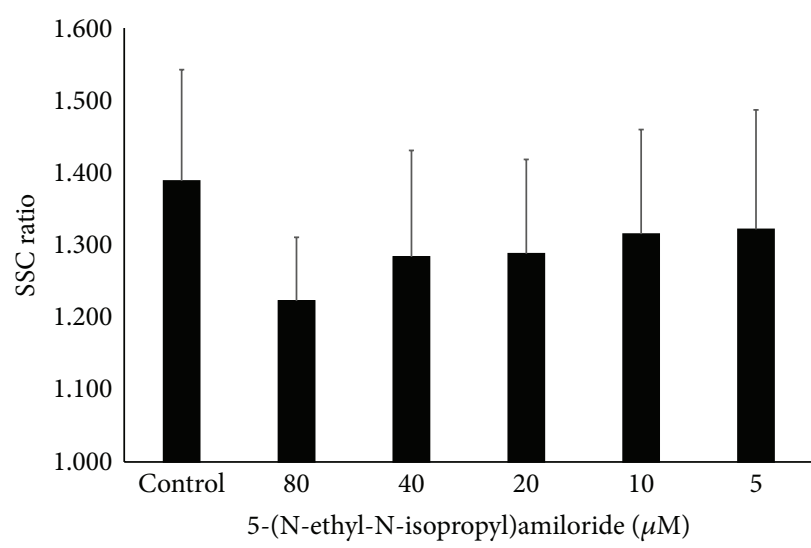

(a)

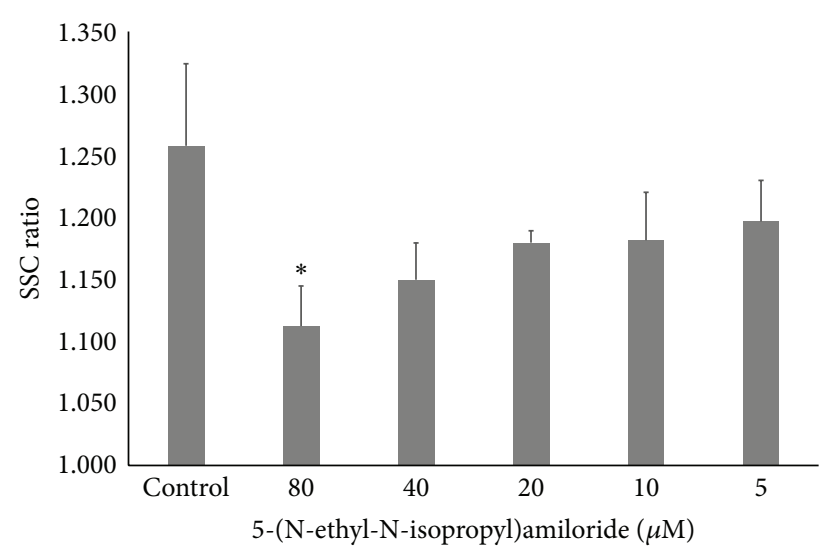

(b)

FIGURE 5: Effect of a macropinocytosis inhibitor on cellular uptake of MWNT-7 cells. The SSC ratios of (a) BEAS-2B cells and (b) MESO1 cells pretreated with various concentrations of 5-(N-ethyl-N-isopropyl)amiloride are shown. The cells were compared with control cells pretreated with inhibitor solvent. Mean \pm SD. $n=3,{ }^{*} P<0.05$.

The latest information for cellular uptake of nanomaterials has been reviewed and suggests that three different mechanisms of endocytosis exist including clathrin- and caveolaeindependent endocytosis, and also endocytosis depends on particle physical-chemical properties, experimental conditions, and cell type in nonphagocytic cells [48]. We consider that CNT uptake is also subject to the same influences in an interdependent manner because the total inhibition rate when independently inhibited pathways were considered together easily exceeded $100 \%$, which means that other pathways function in a compensatory manner, even when one pathway is inhibited. Moreover, other pathways may exist because there are some reports indicating several types of clathrin- and caveolae-independent endocytosis, and the endocytic mechanism is especially unexplained in the nonphagocytic cells [49-51]. In fact, it was not possible to clarify the mechanism underlying the observed suppression of CNT uptake in BEAS-2B cells cultured in FBS-free medium [32]. That study also indicated that the degree of aggregation is an important factor but we could not clarify this issue. We measured the SSC ratio in the comparatively early stage of $2 \mathrm{~h}$ after CNT exposure because high concentrations of the inhibitors showed cytotoxicity. Within $2 \mathrm{~h}$, a small fibrous agglomerate containing some MWCNTs was seen at the bottom of the dish. Although it seems likely that our inhibitor results reflect actual endocytosis, it is unclear whether the nonagglomerated MWCNTs observed after $2 \mathrm{~h}$ at the bottom in Movie S1 and Movie S2 show the same result. However, there appeared to be a common cellular uptake pattern for the MWCNTs.

In conclusion, we found that human normal bronchial epithelial cells and mesothelium cells endocytosed MWCNTs. The mechanism of endocytosis seemed to be not only one but a combination of three pathways: clathrin-mediated endocytosis, caveolae-mediated endocytosis, and macropinocytosis. Although clathrin-mediated endocytosis played the most important role, other pathways may be involved to varying degrees. The cellular uptake of MWCNTs is essential for MWCNT toxicity in the context of genotoxicity. It may thus be necessary to prepare materials that are not endocytosed to develop the nanomaterials having not only useful but also hazardous properties, as we alluded to in a previous study [28]. We have already reported that both BEAS-2B and MESO-1 cells did not endocytose MWCNTs dispersed in carboxymethyl cellulose. Therefore, this and previous studies suggest that biocompatible nanomaterials can be developed.

\section{Conflict of Interests}

The authors declare that there is no conflict of interests regarding the publication of this paper.

\section{Acknowledgments}

The authors would like to thank the staff at the Division of Instrumental Analysis at the Research Center for Human and Environmental Sciences of Shinshu University for their assistance. This research was supported by JSPS KAKENHI Grant nos. 24241045, 25350524, and 25462365; the Center of Innovation Program from Japan Science and Technology Agency, JST; a Health Labour Sciences Research Grant from the Ministry of Health Labour and Welfare, Japan (no. 12103240); and the Hospital-Company Collaboration Support Project for Developing/Improving Problem-Solving-Type Medical Equipment by the Ministry of Economy, Trade and Industry, Japan (nos. 143 and 24-055).

\section{References}

[1] A. Oberlin, M. Endo, and T. Koyama, "Filamentous growth of carbon through benzene decomposition," Journal of Crystal Growth, vol. 32, no. 3, pp. 335-349, 1976.

[2] M. Endo, M. S. Strano, and P. M. Ajayan, "Potential applications of carbon nanotubes," Topics in Applied Physics, vol. 111, pp. 1361, 2008. 
[3] M. F. L. de Volder, S. H. Tawfick, R. H. Baughman, and A. J. Hart, "Carbon nanotubes: present and future commercial applications," Science, vol. 339, no. 6119, pp. 535-539, 2013.

[4] N. Saito, H. Haniu, Y. Usui et al., "Safe clinical use of carbon nanotubes as innovative biomaterials," Chemical Reviews, vol. 114, no. 11, pp. 6040-6079, 2014.

[5] N. Saito, K. Aoki, Y. Usui et al., "Application of carbon fibers to biomaterials: a new era of nano-level control of carbon fibers after 30-years of development," Chemical Society Reviews, vol. 40, no. 7, pp. 3824-3834, 2011.

[6] A. Abarrategi, M. C. Gutiérrez, C. Moreno-Vicente et al., "Multiwall carbon nanotube scaffolds for tissue engineering purposes," Biomaterials, vol. 29, no. 1, pp. 94-102, 2008.

[7] H. Haniu, N. Saito, Y. Matsuda et al., "Basic potential of carbon nanotubes in tissue engineering applications," Journal of Nanomaterials, vol. 2012, Article ID 343747, 10 pages, 2012.

[8] K. J. Siegrist, S. H. Reynolds, M. L. Kashon et al., "Genotoxicity of multi-walled carbon nanotubes at occupationally relevant doses," Particle and Fibre Toxicology, vol. 11, no. 1, article 6, 2014.

[9] A. Erdely, M. Dahm, B. T. Chen et al., "Carbon nanotube dosimetry: from workplace exposure assessment to inhalation toxicology," Particle and Fibre Toxicology, vol. 10, no. 1, article 53, 2013.

[10] K. Donaldson, F. A. Murphy, R. Duffin, and C. A. Poland, "Asbestos, carbon nanotubes and the pleural mesothelium: a review of the hypothesis regarding the role of long fibre retention in the parietal pleura, inflammation and mesothelioma," Particle and Fibre Toxicology, vol. 7, article 5, 2010.

[11] G. Oberdörster, "Safety assessment for nanotechnology and nanomedicine: concepts of nanotoxicology," Journal of Internal Medicine, vol. 267, no. 1, pp. 89-105, 2010.

[12] R. R. Mercer, A. F. Hubbs, J. F. Scabilloni et al., "Distribution and persistence of pleural penetrations by multi-walled carbon nanotubes," Particle and Fibre Toxicology, vol. 7, article 28, 2010.

[13] A. Takagi, A. Hirose, T. Nishimura et al., "Induction of mesothelioma in $\mathrm{p} 53+/-$ mouse by intraperitoneal application of multiwall carbon nanotube," Journal of Toxicological Sciences, vol. 33, no. 1, pp. 105-116, 2008.

[14] Y. Sakamoto, D. Nakae, N. Fukumori et al., "Induction of mesothelioma by a single intrascrotal administration of multiwall carbon nanotube in intact male Fischer 344 rats," Journal of Toxicological Sciences, vol. 34, no. 1, pp. 65-76, 2009.

[15] A. Takagi, A. Hirose, M. Futakuchi, H. Tsuda, and J. Kanno, "Dose-dependent mesothelioma induction by intraperitoneal administration of multi-wall carbon nanotubes in p53 heterozygous mice," Cancer Science, vol. 103, no. 8, pp. 1440-1444, 2012.

[16] H. Nagai, Y. Okazaki, S. H. Chew et al., "Diameter and rigidity of multiwalled carbon nanotubes are critical factors in mesothelial injury and carcinogenesis," Proceedings of the National Academy of Sciences of the United States of America, vol. 108, no. 49, pp. E1330-E1338, 2011.

[17] D. W. Porter, A. F. Hubbs, R. R. Mercer et al., "Mouse pulmonary dose- and time course-responses induced by exposure to multiwalled carbon nanotubes," Toxicology, vol. 269, no. 2-3, pp. 136$147,2010$.

[18] J. P. Ryman-Rasmussen, M. F. Cesta, A. R. Brody et al., "Inhaled carbon nanotubes reach the subpleural tissue in mice," Nature Nanotechnology, vol. 4, no. 11, pp. 747-751, 2009.

[19] R. R. Mercer, J. F. Scabilloni, A. F. Hubbs et al., "Extrapulmonary transport of MWCNT following inhalation exposure," Particle and Fibre Toxicology, vol. 10, no. 1, article 38, 2013.
[20] J. Muller, I. Decordier, P. H. Hoet et al., "Clastogenic and aneugenic effects of multi-wall carbon nanotubes in epithelial cells," Carcinogenesis, vol. 29, no. 2, pp. 427-433, 2008.

[21] N. Kobayashi, M. Naya, M. Ema et al., "Biological response and morphological assessment of individually dispersed multi-wall carbon nanotubes in the lung after intratracheal instillation in rats," Toxicology, vol. 276, no. 3, pp. 143-153, 2010.

[22] H. Ellinger-Ziegelbauer and J. Pauluhn, "Pulmonary toxicity of multi-walled carbon nanotubes (Baytubes) relative to alphaquartz following a single $6 \mathrm{~h}$ inhalation exposure of rats and a 3 months post-exposure period," Toxicology, vol. 266, no. 1-3, pp. 16-29, 2009.

[23] L. Ma-Hock, S. Treumann, V. Strauss et al., "Inhalation toxicity of multiwall carbon nanotubes in rats exposed for 3 months," Toxicological Sciences, vol. 112, no. 2, pp. 468-481, 2009.

[24] J. Muller, M. Delos, N. Panin, V. Rabolli, F. Huaux, and D. Lison, "Absence of carcinogenic response to multiwall carbon nanotubes in a 2-year bioassay in the peritoneal cavity of the rat," Toxicological Sciences, vol. 110, no. 2, pp. 442-448, 2009.

[25] L. M. Sargent, A. A. Shvedova, A. F. Hubbs et al., "Induction of aneuploidy by single-walled carbon nanotubes," Environmental and Molecular Mutagenesis, vol. 50, no. 8, pp. 708-717, 2009.

[26] L. M. Sargent, S. H. Reynolds, and V. Castranova, "Potential pulmonary effects of engineered carbon nanotubes: in vitro genotoxic effects," Nanotoxicology, vol. 4, no. 4, pp. 396-408, 2010.

[27] L. M. Sargent, A. F. Hubbs, S.-H. Young et al., "Single-walled carbon nanotube-induced mitotic disruption," Mutation Research: Genetic Toxicology and Environmental Mutagenesis, vol. 745, no. 1-2, pp. 28-37, 2012.

[28] H. Haniu, N. Saito, Y. Matsuda et al., "Effect of dispersants of multi-walled carbon nanotubes on cellular uptake and biological responses," International Journal of Nanomedicine, vol. 6, pp. 3295-3307, 2011.

[29] T. Tsukahara and H. Haniu, "Cellular cytotoxic response induced by highly purified multi-wall carbon nanotube in human lung cells," Molecular and Cellular Biochemistry, vol. 352, no. 1-2, pp. 57-63, 2011.

[30] T. Tsukahara, Y. Matsuda, Y. Usui, and H. Haniu, "Highly purified, multi-wall carbon nanotubes induce light-chain $3 \mathrm{~B}$ expression in human lung cells," Biochemical and Biophysical Research Communications, vol. 440, no. 2, pp. 348-353, 2013.

[31] M. Endo, "Grow carbon fibers in the vapor phase," ChemTech, vol. 18, no. 9, pp. 568-576, 1988.

[32] H. Haniu, N. Saito, Y. Matsuda et al., "Culture medium type affects endocytosis of multi-walled carbon nanotubes in BEAS2B cells and subsequent biological response," Toxicology in Vitro, vol. 27, no. 6, pp. 1679-1685, 2013.

[33] R. Yumoto, H. Nishikawa, M. Okamoto, H. Katayama, J. Nagai, and M. Takano, "Clathrin-mediated endocytosis of FITCalbumin in alveolar type II epithelial cell line RLE-6TN," The American Journal of Physiology-Lung Cellular and Molecular Physiology, vol. 290, no. 5, pp. L946-L955, 2006.

[34] N. Usami, T. Fukui, M. Kondo et al., "Establishment and characterization of four malignant pleural mesothelioma cell lines from Japanese patients," Cancer Science, vol. 97, no. 5, pp. 387-394, 2006.

[35] H. Haniu, N. Saito, Y. Matsuda et al., "Elucidation mechanism of different biological responses to multi-walled carbon nanotubes using four cell lines," International Journal of Nanomedicine, vol. 6, pp. 3487-3497, 2011. 
[36] H. Haniu, N. Saito, Y. Matsuda et al., "Biological responses according to the shape and size of carbon nanotubes in BEAS2B and MESO-1 cells," International Journal of Nanomedicine, vol. 9, no. 1, pp. 1979-1990, 2014.

[37] N. R. Jacobsen, G. Pojano, H. Wallin, and K. A. Jensen, "Nanomaterial dispersion protocol for toxicological studies in ENPRA," Internal ENPRA Project Report 6, The National Research Centre for the Working Environment, 2010.

[38] S. Hirano, Y. Fujitani, A. Furuyama, and S. Kanno, "Uptake and cytotoxic effects of multi-walled carbon nanotubes in human bronchial epithelial cells," Toxicology and Applied Pharmacology, vol. 249, no. 1, pp. 8-15, 2010.

[39] L. Tabet, C. Bussy, N. Amara et al., "Adverse effects of industrial multiwalled carbon nanotubes on human pulmonary cells," Journal of Toxicology and Environmental Health-Part A: Current Issues, vol. 72, no. 2, pp. 60-73, 2009.

[40] H. K. Lindberg, G. C.-M. Falck, R. Singh et al., "Genotoxicity of short single-wall and multi-wall carbon nanotubes in human bronchial epithelial and mesothelial cells in vitro," Toxicology, vol. 313, no. 1, pp. 24-37, 2013.

[41] K. G. Rothberg, J. E. Heuser, W. C. Donzell, Y.-S. Ying, J. R. Glenney, and R. G. W. Anderson, "Caveolin, a protein component of caveolae membrane coats," Cell, vol. 68, no. 4, pp. 673-682, 1992.

[42] J. A. Allen, J. Z. Yu, R. J. Donati, and M. M. Rasenick, “ $\beta$ adrenergic receptor stimulation promotes $\mathrm{G} \alpha$ s internalization through lipid rafts: a study in living cells," Molecular Pharmacology, vol. 67, no. 5, pp. 1493-1504, 2005.

[43] S. B. Sieczkarski and G. R. Whittaker, "Dissecting virus entry via endocytosis," Journal of General Virology, vol. 83, no. 7, pp. 1535-1545, 2002.

[44] E. J. Smart, K. Estes, and R. G. W. Anderson, "Inhibitors that block both the internalization of caveolae and the return of plasmalemmal vesicles," Cold Spring Harbor Symposia on Quantitative Biology, vol. 60, pp. 243-248, 1995.

[45] C. C. Visser, S. Stevanović, L. H. Voorwinden et al., "Validation of the transferrin receptor for drug targeting to brain capillary endothelial cells in vitro," Journal of Drug Targeting, vol. 12, no. 3, pp. 145-150, 2004.

[46] S. Hirano, Y. Fujitani, A. Furuyama, and S. Kanno, "Macrophage receptor with collagenous structure (MARCO) is a dynamic adhesive molecule that enhances uptake of carbon nanotubes by CHO-K1 Cells," Toxicology and Applied Pharmacology, vol. 259, no. 1, pp. 96-103, 2012.

[47] S. Hirano, S. Kanno, and A. Furuyama, "Multi-walled carbon nanotubes injure the plasma membrane of macrophages," Toxicology and Applied Pharmacology, vol. 232, no. 2, pp. 244-251, 2008.

[48] K. Kettler, K. Veltman, D. van de Meent, A. van Wezel, and A. J. Hendriks, "Cellular uptake of nanoparticles as determined by particle properties, experimental conditions, and cell type," Environmental Toxicology and Chemistry, vol. 33, no. 3, pp. 481492, 2014.

[49] S. D. Conner and S. L. Schmid, "Regulated portals of entry into the cell," Nature, vol. 422, no. 6927, pp. 37-44, 2003.

[50] G. J. Doherty and H. T. McMahon, "Mechanisms of endocytosis," Annual Review of Biochemistry, vol. 78, pp. 857-902, 2009.

[51] I. H. Pastan and M. C. Willingham, "Receptor-mediated endocytosis of hormones in cultured cells," Annual Review of Physiology, vol. 43, pp. 239-250, 1981. 

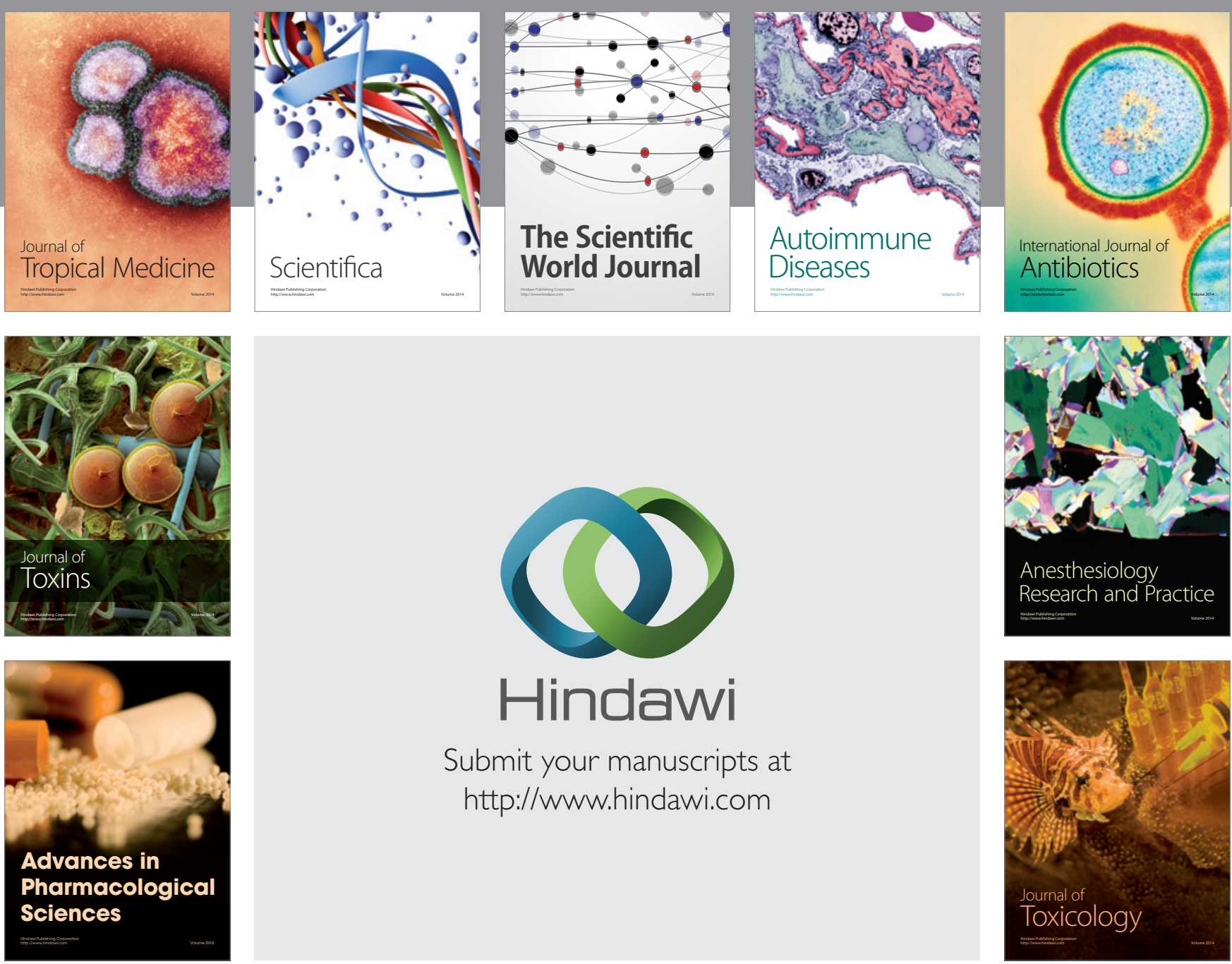

\section{Hindawi}

Submit your manuscripts at

http://www.hindawi.com
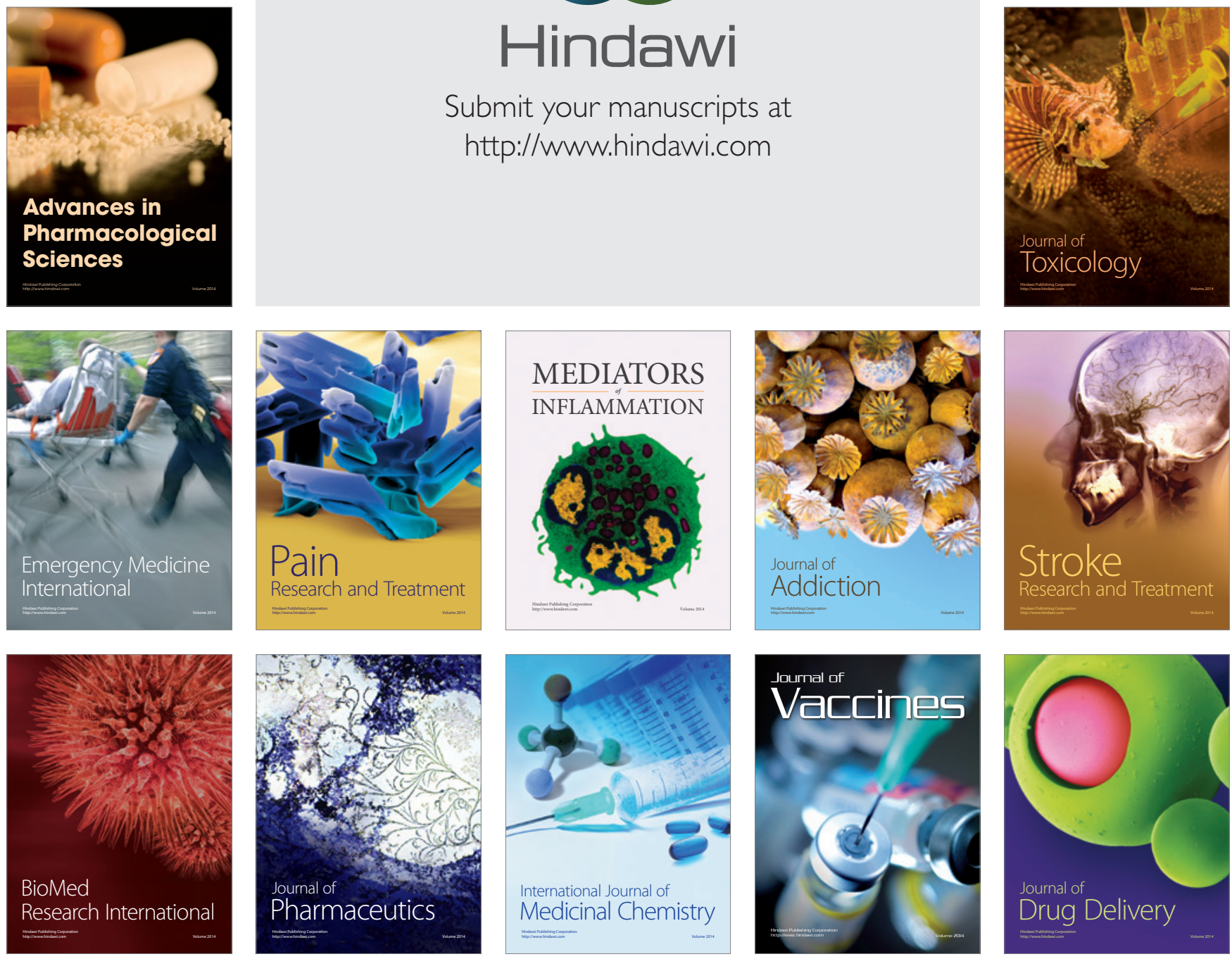